\title{
CA. de Endometrio en Pacientes Jóvenes
}

Dres.: Gabriel Fernando Uribe E. * Gabriel Alberto Tobón L. *
Jaime Garcia M. *

Constanza Diaz de C. **
Hasta hace unos años el $\mathrm{Ca}$. de endometrio en paciente menores de 40 años era una curiosidad médica. Schroder 1 en 1922 informó casos de $\mathrm{Ca}$. de endometrio asociado a tumor de la granulosa y Speert 2 en 1949 informó los primeros 14 casos de $\mathrm{Ca}$. de endometrio en pacientes menores de 40 años. 8 de las cuales presentaban enfermedad poliquística del ovario. Posteriormente se han descrito cuatro grupos de entidades las cuales se asocian con presencia temprana de Ca. endometrial. Estas son:

1. Tumores funcionantes de ovario (granulomas, tecomas, mixtos).

2. Enfermedad poliquistica del ovario.

3. Terapia estrogénica (generalmente de sustitución luego de ooforectomía, en menopáusicas, sindiome de Turner) 3 .

4. Ingestión prolongada de anovulatorios 4 .

Existen otros factores de riesgo: obesidad, esterilidad, hemorragia uterina disfuncional, diabetes, hiperplasia endometrial, inmunosupresores.

En la sección de Oncología, del Servicio de Obstetricia y Ginecologia del Hospital Universitario San Vicente de Paúl, se lleva desde hace 10 años un

\footnotetext{
* Departamento de Obstetricia y Ginecologia

- Universidad de Antioquia.

** Departamento de Patologia - Universidad de Antioquia.
}

registro de las pacientes recibidas con el diagnóstico de $\mathrm{Ca}$. de endometrio. Las pacientes de menor edad fueron 3 , quienes tenían 41 años. Por lo anterior. nos causó gran sorpresa el encontrar en el Servicio de Ginecología del Con. sultorio Departamental del S.S S.A. una paciente de 25 años con $\mathrm{Ca}$. de endometrio, cuya historia presentaremos más adelante. Tal vez el caso más joven presentado en la última década es el de William A. Eddy" quien informa de una paciente de 21 años con $\mathrm{Ca}$. de endometrio asociado con enfermedad poliquística del ovario.

"Nuestra paciente es una maestra de 25 años, soltera, grávida $\mathrm{O}$, sin relaciones sexuales, quien consultó a un hospital de área rural, por presentar severa hemorragia vaginal. La menarquía había sido a los 16 años y sus ciclos eran irregulares con oligomenorrea hasta de 3 meses. No había recibido ningún tipo de terapia hormonal antericrmente a la consulta. En vista del severo sangrado y de que la paciente narraba una amenorrea de dos meses, el médico que la recibió le practicó un curetaje.

Al examen encontró un útero ligera. mente aumentado de tamaño, anexos libres. Extrajo gran cantidad de material endometrial lo cual lo hizo sospechar un aborto incompleto a pesar de la insistencia de la paciente en negar relaciones sexuales. Para salir de dudas, envió el material a estudio, el cual fue informado como $\mathrm{Ca}$. de endometrio, moderadamente bien diferenciado. 
Al ser recibida la paciente en el Consultorio Médico Departamental, se encontró una joven de 1.55 de estatura, 52 $\mathrm{kg}$ de peso, con moderado hirsutismo facial y de miembros inferiores. No obesa. Examen general normal, útero de tamaño normal, $7 \mathrm{~cm}$ de histerometría, móvil, anexos libres, parametrios libres. Se tomó una nueva biopsia de endometrio, también informada como $\mathrm{Ca}$. endometrial.
Se pidieron $\mathrm{FSH}$ y $\mathrm{LH}$, resultando $\mathrm{FSH}$ baja y LH aumentado, tal como se describe en enfermedad poliquística del ovario. 17 cetosteroides aumentados y 17 hidroxiesteroides normales. El resto de prequirúrgicos normales (Cuadro $\mathrm{N}$ : 1). Nos decidimos por la anexo histerectomía, luego de pensar mucho en la terapia hormonal, especialmente por el difícil control de la paciente pues vive en un área rural.

\section{CUADRO № 1}

Resultados de los exámenes de laboratorio

\begin{tabular}{lrc} 
& & Normal \\
17 hidroxiesteroides & $3.4 \mathrm{mg} / 24$ & $9-1$ \\
17 cetosteroides & $18.3 \mathrm{mg} / 24$ & $6-15$ \\
L.H. RIA & $2.8 \mathrm{ng} / \mathrm{ml}$ & $0.9-4.7$ \\
F.S.H. RIA & $1.0 \mathrm{ng} / \mathrm{ml}$ & $1.3-4.5$ \\
P.R.L. RIA & $13.3 \mathrm{ng} / \mathrm{ml}$ & $0-25$ \\
\hline
\end{tabular}

Figura No. 1

FISIOPATOLOGIA DEL CARCINOMA DE ENDOMETRIO EN LA ENFERMEDAD POLIOUISTICA DEL OVARIO

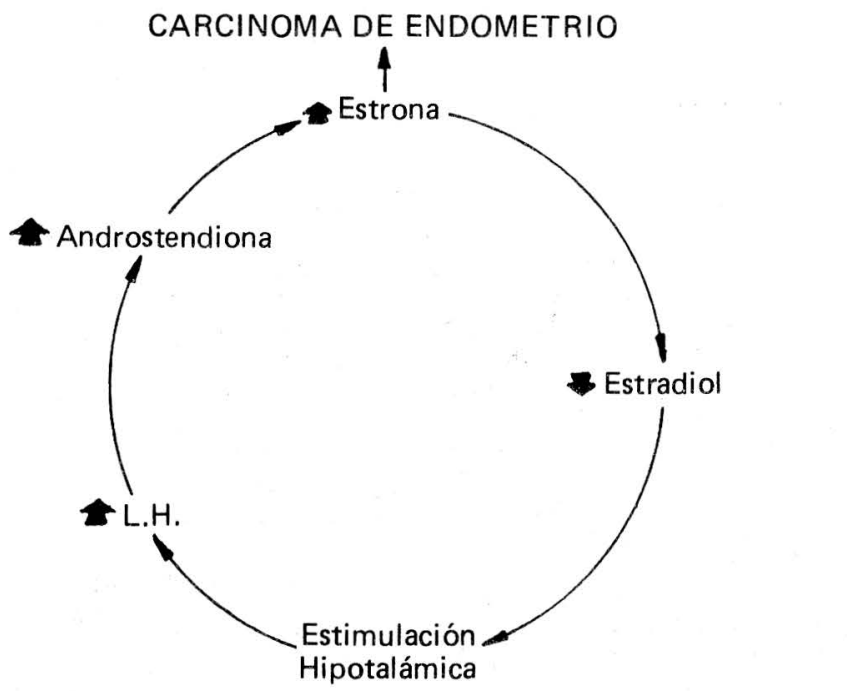


La cirugía se practicó sin complicaciones intra y post-operatorias. El resultado de la pieza quirúrgica aún mostraba $\mathrm{Ca}$ de endometrio (Fig. 2).
Actualmente recibe terapia de sustitución, lo cual puede ser discutible, pero de vital importancia erı una paciente tan joven'.

Figura No. 2

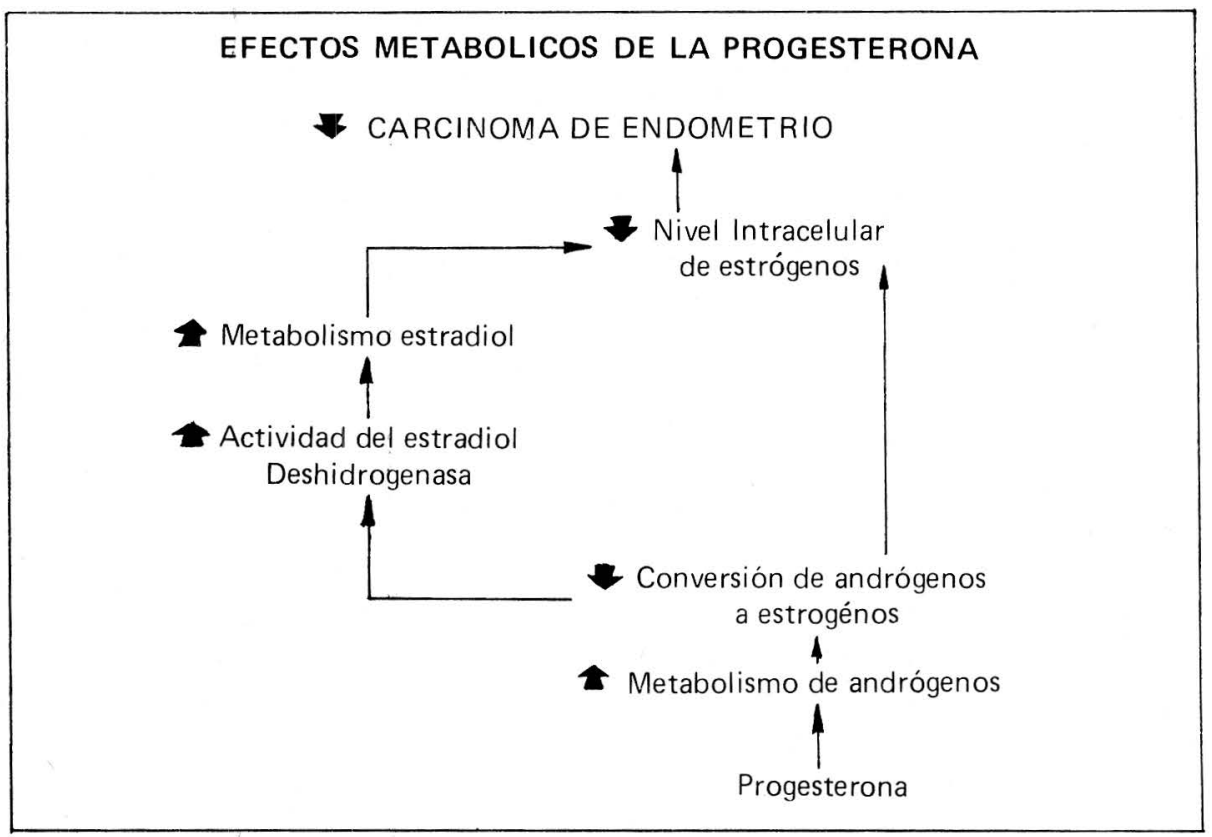

\section{Comentarios}

Es evidente que el ginecólogo debe pensar más ahora en el Ca. de endometrio en pacientes menores de 40 años, especialmente si estas pacientes se clasifican en uno de los 4 grupos de riesgo descritos anteriormente. Al parecer la estimulación crónica del ovario inducida por altos niveles de LH en la enfermedad poliquística, trae como consecuencia la producción de androstendiona. Este disbalance hormonal parece ser un factor causal en el desarrollo de carcinoma de endometrio 6 . La exclusiva producción de estrógenos en pacientes con anovulación está asociada a un altc riesgo de $\mathrm{Ca}$. de endometrio y de mama 7 (Figura $N$ ․ 1). Por otro lado, la progesterona aumenta el metabo!ismo de los andrógenos, reduciendo la conversión de estrógenos a andrógenos, aumenta la actividad del estradiol des. hidroginasa del endometrio, acelerando el metabolismo del estradiol y disminuyendo los niveles intracelulares de estrógenos. Esto explica los efectos protectores antiestrogénicos de la progesterona y su valor terapéutico en el $\mathrm{Ca}$. de endometrio. Sin embargo, existen criterios dispares entre la utilidad de tratar pacientes jóvenes con $\mathrm{Ca}$. de endometrio con altas dosis de proçesterona, como lo sugieren Fechner y Kauf- 
man 8 o tratarlas como cualquier otro $\mathrm{Ca}$. de endometrio, como lo afirman Jafari y col 6 (Cuadro $N: 2$ y Fig. № 2).

\section{CUADRO $\mathrm{N} \div 2$}

\section{Tratamiento médico}

(A.)

1. Caproato de 17 a hidroxiprogesterona

Dosis: $1.000 \mathrm{mg} 3$ veces por semana durante un mes.

2. Medroxiprogesterona Dosis: $10 \mathrm{mg}$ del día 15 al 25 del ciclo.

(B.)

\section{Acetato de medroxiprogesterona}

Dosis: 1 gramo semanal por 3 meses

Existe sí uniformidad en cuanto a la lesión microscópica y al pronóstico. Cerca del $90 \%$ de los adenocarcinomas de endometrio asociado a la enfermedad poliquística del ovario son bien diferenciados y su pronóstico es excelente. Io mismo sucede en la asociación con tumores funcionantes de ovario 9 y al parecer, en los $\mathrm{Ca}$. de endometrio encontrados en pacientes que toman anticonceptivos 4 .

Por último, no encontramos un solo concepto en cuanto al uso o no de terapia de sustitución en pacientes sometidas a cirugía o a radioterapia por su $\mathrm{Ca}$. de endometrio. Creemos que este aspecto vale la pena de ser más estudiado.

\section{Resumen}

Hasta hace unos pocos años, el carcinoma de endometrio en pacientes menores de 40 años era una curiosidad médica.
Se hace una revisión de las causas predisponentes entre las cuales se describen:

1. Tumores funcionantes de ovario

2. Enfermedad poliquística del ovario

3. Terapia estrogénica

4. Ingestión prolongada de anovulatorios.

Se presenta el caso de una paciente de 25 años, vista en el Consultorio Miédico Departamental, a quien se le prac. ticó anexo histerectomía por un $\mathrm{Ca}$. de endometrio. Se discute el estudio anatomopatológico.

Se hace un análisis de la influencia de los estrógenos en el carcinoma de endometrio

Se discute el tratamiento comparando el médico y el quirúrgico.

Por último se deja el interrogante sobre terapia de sustitución en estas pacientes.

\section{Endometrium carcinoma in young patients}

\section{Summary}

Until recent years the Endometrium Carcinoma in patients of less than 40 years old was a medical inquisitiveness.

A revision is made of the predisposing causes, of which the following are described:

1. Ovarian functioning tumors

2. Ovarian polycystic illness

3. Estrogenic therapy

4. Prolonged anovulatory ingestion

A case of a 25 years old patient nursed at the Departmental Medical Consulting Room and submitted to an Anexo-Hysterectomy due to Endometrium Carcinoma, is presented. The anatomo-pathological stury is discussed. 
An anaysis is made of the influence of estrogens in the Endometrium Carcinoma.

The treatment is discussed by comparing the medical and the surgical treatment.

Finally, the therapy of substitution in these patients is questioned.

\section{Bibliografía}

1. SCHRADER, R. Granulosazelltumor des ovars mit Glandularzystischer Hyperplasies des Endometriams und beginnendem Karzinom and diesem Bodem. Monatsscher Geburts hilfe Gunaekol. 58: 294-295, 1922.

2. SPEERT, H. Carcinoma of the endometriom in young women Surg. Obstet Gynecol 88: 332-336, 1949.

3. GRAY, L. A. Christopherson, W. M., HOOVER, R. N.: Estrones and Endometrial Carcinoma Obstet Gynecol 49: 385-389, 1977.
4. LYON, F. A. Am. J. Obstet Gynecol 123: 299, 1975.

5. EDDY, W. A.: Endometrial Carcinoma in Stein - Leventhal oynchome treated with hydroxyprogesterona caproate Am. J. Obstet Gynecol 131: 581-582, 1978.

6. JAFARI, K., JAVAHERI, G., RUIZ, G.: Endometrial adenocarcinoma y sindrome de Stein Leventhal Obstet Gynecol 51: 97-99, 1978.

7. SITTER: PK, SCHWARZ B. E., MACDONALD, P. C.: Estrogen receptors and the estrone hypothesis in relation to endometrial and Breast. Cancer Gynecol Oncol 2: 228-238, 1974.

8. FECHNER, R. F., KAUFMAN, R. M.: Endometrial adenocarcinoma and Polycistic ovary sindrome Am. J. Obstet Gynecol 124: 140-142, 1976.

9. MAC DONALD E. W. MAL KASIAN, G. D., GOFFEY A.: Endometrial cancer associated with feminizing ovarian Tumor and polycistic ovarian disease. 